\title{
Hemodynamic response function (HRF) variability confounds resting-state fMRI functional connectivity
}

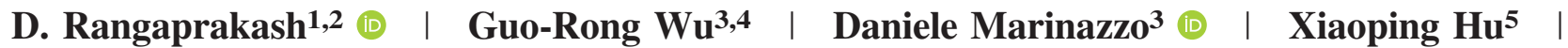 \\ Gopikrishna Deshpande ${ }^{1,6,7,8}$ (D)}

${ }^{1}$ Auburn University MRI Research Center, Department of Electrical and Computer Engineering, Auburn University, Auburn, Alabama

${ }^{2}$ Department of Psychiatry and Biobehavioral Sciences, University of California Los Angeles, Los Angeles, California

${ }^{3}$ Department of Data Analysis, University of Ghent, Ghent, Belgium

${ }^{4}$ Key Laboratory of Cognition and Personality, Southwest University, Chongqing, China

${ }^{5}$ Department of Bioengineering, University of California Riverside, Riverside, California

${ }^{6}$ Department of Psychology, Auburn University, Auburn, Alabama

${ }^{7}$ Center for Health Ecology and Equity Research, Auburn University, Auburn, Alabama

${ }^{8}$ Alabama Advanced Imaging Consortium, Auburn University, University of South Alabama and University of Alabama at Tuscaloosa and Birmingham, Alabama

\section{Correspondence}

Gopikrishna Deshpande, 560 Devall Dr., Suite 266D, Auburn University, Auburn, AL 36849, USA.

Email: gopi@auburn.edu;

Twitter: @gopideshpande
Purpose: fMRI is the convolution of the hemodynamic response function (HRF) and unmeasured neural activity. HRF variability (HRFv) across the brain could, in principle, alter functional connectivity (FC) estimates from resting-state fMRI (rs-fMRI). Given that $\mathrm{HRFv}$ is driven by both neural and non-neural factors, it is problematic when it confounds FC. However, this aspect has remained largely unexplored even though FC studies have grown exponentially. We hypothesized that HRFv confounds FC estimates in the brain's default-mode-network.

Methods: We tested this hypothesis using both simulations (where the ground truth is known and modulated) as well as rs-fMRI data obtained in a 7T MRI scanner $(N=47$, healthy). FC was obtained using 2 pipelines: data with hemodynamic deconvolution (DC) to estimate the HRF and minimize HRFv, and data with no deconvolution (NDC, HRFv-ignored). DC and NDC FC networks were compared, along with regional HRF differences, revealing potential false connectivities that resulted from HRFv.

Results: We found evidence supporting our hypothesis using both simulations and experimental data. With simulations, we found that HRFv could cause a change of up to $50 \%$ in FC. With rs-fMRI, several potential false connectivities attributable to HRFv, with majority connections being between different lobes, were identified. We found a double exponential relationship between the magnitude of HRFv and its impact on FC, with a mean/median error of $30.5 / 11.5 \%$ caused in FC by HRF confounds.

Conclusion: HRFv, if ignored, could cause identification of false FC. FC findings from $\mathrm{HRFv}$-ignored data should be interpreted cautiously. We suggest deconvolution to minimize HRFv.

\section{K E Y W O R D S}

deconvolution, functional connectivity, functional magnetic resonance imaging, hemodynamic response function, HRF variability 


\section{1 | INTRODUCTION}

fMRI is a popularly used technique for studying neural correlates of brain functioning. However, one limitation of fMRI is that it is not a direct measure of neural activity, because it measures changes in blood oxygenation level, which is merely modulated by neural activity. Blood oxygenation is also modulated by the vascular structure, hematocrit, and neurochemicals, which couple neural activity with blood flow, volume, and oxygenation. The mathematical transfer function between neural activity and its corresponding blood oxygenation level-dependent (BOLD) fMRI signal is called the hemodynamic response function (HRF). It is dependent on cerebrovascular reactivity and neurovascular coupling. ${ }^{1}$ Most studies assume a standard whole-brain canonical HRF during analysis (typically made of 2 gamma functions), although previous works show HRF variability for different brain regions and across subjects. ${ }^{2-5}$ The variability of non-neural components of HRF across the brain as well as across individuals $^{2,3}$ is problematic. Since only neural activity is of interest in most fMRI studies, interpretation of fMRI findings is often clouded because of the aforementioned non-neural sources of variability in fMRI. This makes the interpretation of fMRI results challenging as it would be unclear as to whether the observed changes are due to neural activity or HRF variability or a combination of the two.

A recent article debating cellular neuroscience's viewpoints on BOLD PMRI $^{6}$ presented numerous caveats while interpreting fMRI results, which demands careful consideration based on the underlying cellular mechanisms. They comment on neurovascular dynamics or HRF variability, which is one such issue, as follows: "advances in cellular neuroscience, demonstrating differences in this neurovascular relationship in different brain regions, conditions or pathologies are often not accounted for when interpreting BOLD." They suggest the community use computational modeling (e.g., deconvolution) to mitigate the issue. Our work attempts to advance these findings by studying the impact of HRF variability on fMRI functional connectivity (FC) and by illustrating how computational modeling such as deconvolution could mitigate the issue.

The human brain's capacity is largely attributable to its high interconnectedness; hence, the study of brain connectivity has gained enormous importance over the last decade. The most widely studied phenomenon of FC measures coactivation of pairs of brain regions. Although HRF variability is known to influence the fMRI signal, ${ }^{2}$ a systematic study of its impact on FC has not emerged. In this work, we study the effect of HRF variability on fMRI FC in the default-mode network $(\mathrm{DMN})^{7}$ of the brain. Our study assumes importance given that the number of fMRI FC studies have been growing exponentially (1535 publications in 2016, and 1994 publications in 2017), yet most of them ignore this variability caused by hemodynamics.

One can classify the variability of the HRF into 3 main classes: (1) intra-subject variability (HRF difference across different brain regions in the same individual), (2) intra-group inter-subject variability (HRF difference across different healthy individuals, for a given location in the brain), and (3) inter-group variability (HRF difference between a healthy and a pathological group for a given location in the brain, arising partly because of vascular and neurochemical disturbances owing to pathology). Each of these could potentially cause misleading results during fMRI data analysis. Intra-subject variability could lead to misclassifying true strong connectivities as weak, and true weak connectivities as strong. HRF variability's effect on activation analysis could be alleviated, in part, by using time and dispersion derivatives in the general linear model. ${ }^{8}$ Although much attention has been received on the effect of HRF variability on lag-based effective connectivity models, ${ }^{9,10}$ its effect on zero-lag FC models has not been explored. We address this issue by investigating the effect of intra-subject HRF variability on zero-lag FC analysis. Specifically, we investigated the effect of intra-subject HRF variability on resting-state FC between default mode network (DMN) regions in healthy young adults.

We hypothesized that the variability in HRF, which depends on both neural and non-neural factors (such as hematocrit, variable density, and size of vasculature, lipid/alcohol/ caffeine ingestion, global magnetic susceptibilities, partial volume imaging of veins, pulse/respiration differences, and slice timing differences), ${ }^{2,3,11,12}$ causes alterations of nonneural origin in DMN FC in healthy adults. We tested this hypothesis using both simulations (where the ground truth is known and can be modulated) as well as experimental data. The latter part was achieved by obtaining connectivity differences between HRF-variability-affected data (i.e., hemodynamic deconvolution not performed) and HRF-variabilityreduced data (i.e., deconvolution performed). When FC is estimated in HRF-deconvolved data, the inferences are based on latent neural variables. These variables, much like in dynamic causal modeling (DCM), ${ }^{13}$ are likely to reflect only neural activity and hence will not be confounded by nonneural factors driving HRF variability. Subsequently, we identified the subset of those connections whose FC estimates obtained in the BOLD and latent neural space were significantly different. We then associated HRF differences between the corresponding pairs of regions with the FC alterations in the paths connecting them. Such connections represent the negative effect of $\mathrm{HRF}$ variability on $\mathrm{FC}$ analysis.

HRF is chiefly characterized by 3 parameters ${ }^{2,3}$ (Figure 1): (1) response height $(\mathrm{RH})$, (2) full-width at half-max (FWHM), and (3) time-to-peak (TTP). Recent works show that altered RH, FWHM, and TTP indicate differing metabolism and microvasculature among others. ${ }^{14}$ Let us consider 


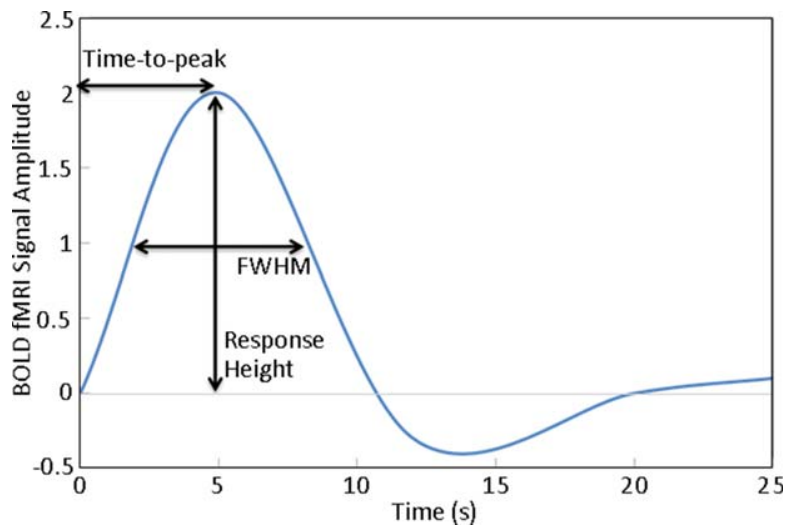

FIGURE 1 Typical hemodynamic response function with its three parameters. FWHM, full-width at half max

the impact of HRF variability on FC analysis. We illustrate 2 possibilities using an example (Figure 2): (1) there could be true high correlation between latent neural signals, but BOLD fMRI time series could show low correlation owing to different TTP delays of the two HRFs, and (2) there could be low correlation between latent neural signals, but BOLD fMRI time series could show high correlation caused by different TTP delays of the two HRFs. The former leads to false-negatives whereas the latter leads to false-positives in traditional fMRI FC analyses that ignores HRF variability. Similar to the example, we sought to find false-negative and false-positive connectivities arising from traditional FC analysis, which ignores HRF variability. Our hypothesis could then be stated that, owing to HRF variability, data without deconvolution would potentially show misleading connectivity differences (both false-positives and false-negatives) as compared to data with deconvolution.
It should be noted that we do not have access to ground-truth HRFs across the brain in experimental data. With current technologies, it is difficult to obtain true HRFs at every voxel in the brain. Region-specific HRFs used in this work are an estimate, obtained in a data-driven way based on biological constraints and mathematical concepts. Hence, we do not take the leap of naming the identified connectivity differences between raw and deconvolved fMRI data as false-positives or falsenegatives; rather we call them pseudo-positives and pseudo-negatives, respectively. Nonetheless, we expect our simulation results, based on known ground truths, to give us more confidence about the type I and type II errors introduced in experimental FC by HRF variability.

The next section presents the methods used to test our hypothesis including the underlying theory, simulations, data pre-processing, connectivity analysis, and HRF analysis.

\section{2 | METHODS}

\section{1 | Theory}

To illustrate the analytical relationship between HRF variability and FC, we first present the theoretical foundations. Pearson's correlation is widely used to quantify FC. The concept underlying our hypothesis is that correlations that exist in latent neuronal data might cease to exist in BOLD fMRI because of different HRFs (and vice versa). This concept can be clearly explained through analytical forms as follows. First, BOLD fMRI is a convolution of latent neuronal time series and the HRF:
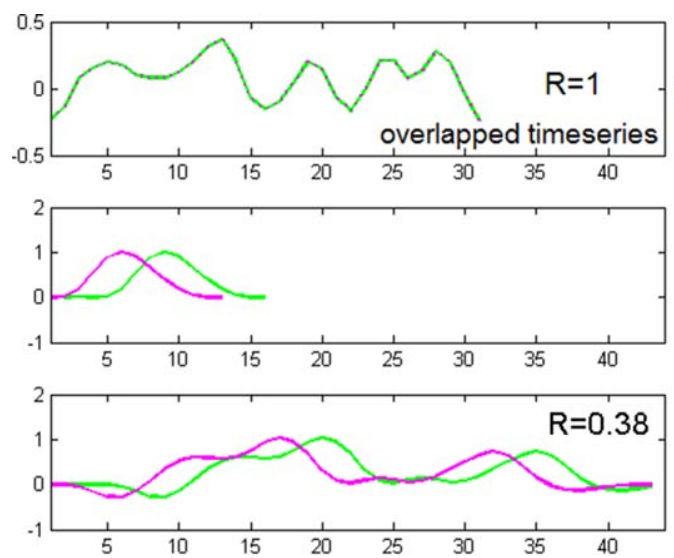

(A)
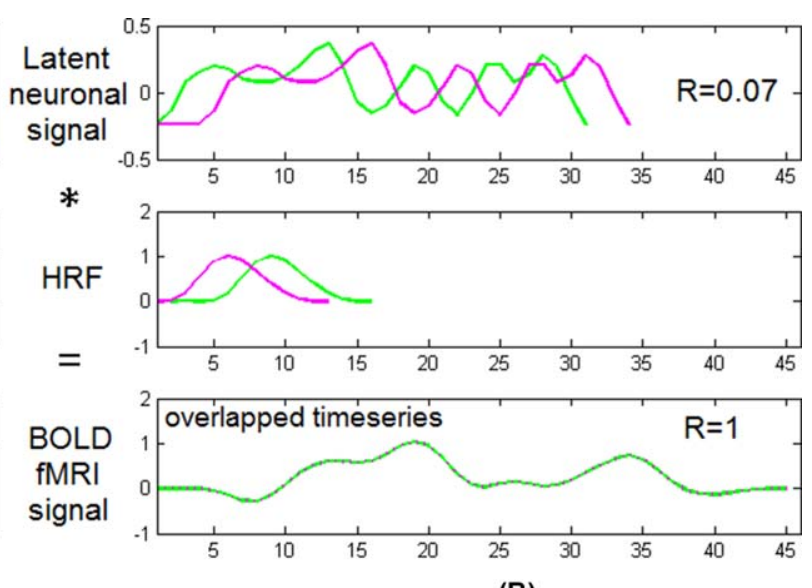

(B)

F I G U RE 2 Illustrating the effect of HRF variability on connectivity analysis. Using a pair of example time series, we demonstrate that: (A) the underlying neural signals are highly correlated while the BOLD fMRI time series are not (giving false low correlation when the true neural correlation is high), leading to pseudo-negatives, which is caused because of different times-to-peak of the HRFs, and (B) the BOLD fMRI time series are highly correlated whereas the underlying neural signals are not (giving false high correlation when the true neural correlation is low), leading to pseudo-positives. This is caused because the HRF corresponding to the leading neuronal time series lags the HRF corresponding to the lagging neuronal time series by the same amount, causing the delays to cancel out and give a high fMRI correlation 


$$
\begin{aligned}
{[\text { BOLD fMRI }]=[} & {[\text { latent neuronal times series }] *[\mathrm{HRF}] } \\
& +[\text { measurement noise }] \\
& \text { or } \mathrm{F}=\mathrm{L} * \mathrm{H}+\mathrm{N},
\end{aligned}
$$

where * is the mathematical convolution operator. Expanding the convolution gives:

$F(k)=\sum_{m=-\infty}^{\infty} L(k-m) H(m)+N(k)=\sum_{m=-\infty}^{\infty} L(m) H(k-m)+N(k)$,

where $F(k)$ is the fMRI time series value at time point $k, L$ is the latent neural variable, $H$ is the HRF, $N$ is measurement noise, and the summation is over all time points $m$. From this equation, it is clear that a delay $d$ in fMRI time series $F$ would be caused by an equal delay $d$ in HRF $H$ as follows:

$$
F(k+d)=\sum_{m=-\infty}^{\infty} L(m) H(k+d-m)+N(k+d) .
$$

Similarly, a delay in latent neuronal time series $L$ would propagate exactly onto the fMRI time series $F$. This property owes to the fact that convolution is a linear time-invariant operation. Therefore, 2 different HRFs result in 2 fMRI time series with differing delays in relation to the latent neuronal time series.

Pearson's correlation is often used to evaluate FC. Given 2 time series $x=[x 1, x 2, \ldots, x T]$ and $y=[y 1, y 2, \ldots, y T]$ (where $T$ is the number of time points), $\mathrm{FC}$ is defined as:

$$
F C=\frac{\sum_{i=1}^{T}\left[\left(x_{i}-\bar{x}\right)\left(y_{i}-\bar{y}\right)\right]}{\sqrt{\sum_{i=1}^{T}\left(x_{i}-\bar{x}\right)^{2}} \sqrt{\sum_{i=1}^{T}\left(y_{i}-\bar{y}\right)^{2}}} .
$$

Here, $\bar{x}$ and $\bar{y}$ are sample means of time series $x$ and $y$. Given that Pearson's correlation is a zero-lag measure, different delays in the 2 time series under consideration caused by different HRFs would mathematically result in different correlation values. Analytically, the resulting correlation between the 2 fMRI time series $x$ and $y$ would be:

$$
F C=\frac{\sum_{i=1}^{T}\left[\left(x_{i+d 1}-\bar{x}\right)\left(y_{i+d 2}-\bar{y}\right)\right]}{\sqrt{\sum_{i=1}^{T}\left(x_{i+d 1}-\bar{x}\right)^{2}} \sqrt{\sum_{i=1}^{N}\left(y_{i+d 2}-\bar{y}\right)^{2}}},
$$

where fMRI time series $x$ is delayed by $d 1$ because of its HRF time-to-peak of $d 1$, and time series $y$ is delayed by $d 2$ because of its HRF time-to-peak of $d 2$. Clearly, the correlation value obtained with different delays would be different from the correlation value obtained with same delays (if $d 1=d 2$ ). Therefore, it is clear from these analytical expressions that delays in HRFs cause equal amount of delays in fMRI time series, and different delays in 2 HRFs result in altered correlation value between the corresponding 2 time series. We explore the implication of these observations on FC in simulated as well as experimental fMRI data.

\section{2 | Simulations}

We performed simulations to illustrate the empirical relationship between HRF parameters and FC. Briefly, we simulated pairs of neural time series with known neural-FC between them, then simulated pairs of HRFs with known difference in HRF parameters between them, then convolved them to obtained pairs of fMRI time series and the fMRI-FC between them.

This simulation technique was adopted from Deshpande et al. ${ }^{15}$ We first simulated pairs of neural time series with a known neural-FC. Two time series, $x(n)$ and $y(n)$ from interacting neuronal populations $X$ and $Y$, respectively, were generated using a first order vector autoregressive (VAR) model with an absence of lagged relationships between $x(n)$ and $y(n)$ and a covariance matrix $C$. The offdiagonal elements of $C$, indicating the cross-correlation between $x(n)$ and $y(n)$, were varied to simulate different functional connectivity strengths between $x(n)$ and $y(n)$. As in previous studies, ${ }^{15}$ we assumed that $x(n)$ and $y(n)$ correspond to local field potentials (LFP) sampled at $1 \mathrm{~ms}$. Next, we simulated pairs of HRFs with known difference in HRF parameters between them, using a commonly used HRF defined from 2 gamma functions as in statistical parametric mapping (SPM) toolbox. ${ }^{16}$ The HRF pairs were generated in such a way that they either differed in the response height $(\Delta \mathrm{RH})$, or the time-to-peak $(\Delta \mathrm{TTP})$ or the full-width at half-max $(\Delta \mathrm{FWHM})$, to independently assess the impact of variability in each of them on FC. Next, we convolved the neural time series with the HRFs and downsampled TR*1000 times to obtain the corresponding fMRI time series pairs, $x^{\prime}(n)$ and $y^{\prime}(n)$. This procedure follows from the currently accepted relationship between LFPs and fMRI. ${ }^{17,18}$ The correlation between them was computed to obtain their fMRI-FC $\left(F C^{f M R I}\right)$. The percentage difference (denoted as $\triangle F C$ ) between neural-FC $\left(F C^{\text {neural }}\right)$ and fMRI$\mathrm{FC}(\triangle \mathrm{FC}$ is the error because of HRF variability) was computed as follows.

$$
\Delta F C=\frac{\left|F C^{\text {neural }}-F C^{f M R I}\right|}{\left|F C^{\text {neural }}\right|} \times 100=\frac{\left|F C^{D C}-F C^{N D C}\right|}{\left|F C^{D C}\right|} \times 100 .
$$

In the experimental data described later, we denote neural-FC as $F C^{D C}$ and fMRI-FC as $F C^{N D C}$, with DC and NDC referring to deconvolved and non-deconvolved data respectively, consistent with the notion that deconvolution of fMRI time series provides latent neural time series.

$\triangle \mathrm{FC}$ was compared with the difference in HRF parameters $(\Delta \mathrm{RH}, \Delta \mathrm{TTP}$, and $\Delta \mathrm{FWHM})$, separately, to obtain 
graphs depicting the relationship between HRF variability and error in FC. The difference in HRF parameters were obtained as follows.

$$
\begin{gathered}
\Delta R H=\frac{\left|R H_{1}-R H_{2}\right|}{\left(R H_{1}+R H_{2}\right) / 2} \times 100 \%, \\
\Delta T T P=\left|T T P_{1}-T T P_{2}\right| s, \text { and } \\
\Delta F W H M=\left|F W H M_{1}-F W H M_{2}\right| s,
\end{gathered}
$$

where the subscripts 1 and 2 correspond to the 2 regions under consideration for which the FC was computed. It must be noted that all the entities in Eqns. (5-9) are absolute values. We varied the value of the off-diagonal elements of $C$ (neural-FC) from -1 to 1 in steps of 0.25 , to obtain the impact of HRF variability at different FC strengths. We varied $\Delta \mathrm{RH}$ from $0 \%$ to $100 \%$ (i.e., percentage change in $\mathrm{RH}$ ) in steps of $0.25 \%$, varied $\Delta \mathrm{TTP}$ from $0-4 \mathrm{~s}$ and $\Delta \mathrm{FWHM}$ from $0-1 \mathrm{~s}$, both in steps of $0.25 \mathrm{~s}$. The absolute value of TTP ranged from 2.5-6.5 s, and that of FWHM ranged from 1-2 s. These values were directly taken from biologically meaningful lower and upper bounds of these parameters in healthy individuals as presented in Handwerker et al. ${ }^{2}$ Additionally, we varied the TR from $0.5-2$ in steps of 0.5 . The simulation was iterated over 10,000 realizations of $x(n), y(n)$, $x^{\prime}(n)$, and $y^{\prime}(n)$. Additional simulations were performed to demonstrate the construct validity of the deconvolution technique. Corresponding details can be obtained from the Supporting Information (section S1).

\section{3 | Resting-state fMRI data}

Forty-seven healthy young adults were recruited for the study. Participants were scanned in a 7T MAGNETOM scanner (Siemens Healthcare, Erlangen, Germany) using $\mathrm{T}_{2}^{*}$-weighted multiband EPI sequence ${ }^{19}$ in resting-state (participants were asked to keep their eyes open and not think of anything specific), with $\mathrm{TR}=1000 \mathrm{~ms}, \mathrm{TE}=20 \mathrm{~ms}$, flip angle $=70^{\circ}$, multiband factor $=2$, voxel size $=2 \times 2 \times$ $2.4 \mathrm{~mm}^{3}$, acquisition matrix $=96 \times 96$, number of slices $=$ 45 and 660 volumes (11 min), with whole-brain coverage. A 32-channel head coil (Nova Medical) was used. The participants provided informed consent and all procedures were approved by the Auburn University Institutional Review Board (IRB).

\section{4 | fMRI data pre-processing}

Standard pre-processing of resting-state fMRI data was performed (slice timing correction with consideration given to the fact that the data was acquired using a multiband sequence, realignment and unwrap, co-registering to anatomical image, de-spiking, normalization to MNI space, spatial smoothing using an $8 \mathrm{~mm}$ Gaussian kernel, regressing out nuisance covariates (6 head motion parameters, Legendre polynomials of orders up to 2nd, top 5 principle components from subject specific white matter [WM] signal and cerebrospinal fluid $[\mathrm{CSF}]$ signal), and band-pass filtering [0.008-0.1 Hz]). Preprocessing was performed on the MATLAB R2013a platform (The MathWorks, Natick, MA) using Statistical Parametric Mapping (SPM12). ${ }^{16}$

To extract the default mode network (DMN) regions of interest (ROIs), we used a popular template provided by Power et al., ${ }^{20}$ which consisted of 58 DMN ROIs (see Supporting Information Table S2 for details). Mean fMRI time series were then obtained from each ROI. The time series data were then subjected to 2 separate pipelines, one with no further pre-processing (contaminated by HRF variability), and another with blind hemodynamic deconvolution (HRF variability largely minimized).

With deconvolution, we obtained latent neuronal variables using a recently reported method. ${ }^{21}$ The method also provided the 3 HRF parameters (RH, TTP, and FWHM). This deconvolution is considered blind because only 1 variable is known (fMRI time series), and from it one estimates both the latent neural time series and the HRF. We used the method proposed by $\mathrm{Wu}$ et al., ${ }^{21}$ which has gained increasing popularity and acceptance owing to its interpretability, robustness, simplicity, validity, and an increasing awareness on the importance of deconvolution. Many recent works have used this method. ${ }^{5,22-29}$ Briefly, the method relies on modeling resting-state fMRI data using point processes as event-related time series with randomly occurring events. ${ }^{30,31}$ A temporal mask with frame-wise displacement $(\mathrm{FD})<0.3$ was added to avoid pseudo point process events induced by motion artifacts. ${ }^{30}$ The HRF was then estimated using Wiener deconvolution. Simulations demonstrating the construct validity of the adopted deconvolution technique are presented in the Supporting Information (section S1). The deconvolution code (on MATLAB platform) was created by Wu et al. ${ }^{21}$ and is available for download. ${ }^{32}$ The HRF parameters obtained have been made freely and publicly available. ${ }^{33}$ All data analysis after pre-processing was performed on the MATLAB R2014a platform.

\subsection{Connectivity analysis}

Time series from 58 DMN regions were obtained in the previous step with data from 2 pipelines: (1) non-deconvolved (NDC) data (HRF-variability-ignored), and (2) deconvolved (DC) data (HRF-variability-reduced). With NDC, FC was evaluated between all pairs of regions to obtain $58 \times 58$ connectivity matrix $\left(F C^{N D C}\right)$ for every participant. Like in most studies, $z$-scored Pearson's correlation coefficient was used to evaluate FC.

Similar procedure was followed with DC data to obtain a $58 \times 58 F C^{D C}$ matrix per participant. The $F C^{N D C}$ and $F C^{D C}$ 
connectivity data have been made publicly available. ${ }^{33}$ Because the difference between $F C^{N D C}$ and $F C^{D C}$ is the deconvolution step and hence the corresponding effect of HRF variability, we sought to identify significant group differences between them. We used a 2-tailed paired t-test to find significant group differences between NDC and DC connectivity matrices $(P<0.05$, Bonferroni corrected). Paired test was chosen because we were looking at withinsubject variability (i.e., connectivities altered in the same participant because of HRF variability), because NDC and DC data are essentially from the same participants. The identified significant differences were then used in further processing.

\subsection{HRF analysis}

Deconvolution provided the estimated HRF for each ROI in each participant. The HRF for each ROI was characterized by 3 parameters: response height $(\mathrm{RH})$, full-width at halfwax (FWHM) and time-to-peak (TTP), as illustrated earlier. That is, we obtained a $58 \times 1$ matrix per participant for $\mathrm{RH}$ $\left(\mathrm{HRF}^{\mathrm{RH}}\right)$, as also for FWHM $\left(\mathrm{HRF}^{\mathrm{FWHM}}\right)$ and TTP $\left(\mathrm{HRF}^{\mathrm{TTP}}\right)$. Then, significant differences in HRF parameters were obtained between all 58 pairs of regions. $\mathrm{HRF}^{\mathrm{RH}}$ for all participants was taken $(58 \times 47$ matrix $)$ and a $P$-value of separation was obtained between every pair of ROIs. This would provide the statistical separation in $\mathrm{HRF}^{\mathrm{RH}}$ between every pair of ROIs (similar with $\mathrm{HRF}^{\mathrm{FWHM}}$ and $\mathrm{HRF}^{\mathrm{TTP}}$ ). We performed 2-sided paired t-test between HRF parameters of all pairs of brain regions $(P<0.05$, Bonferroni corrected). Like with connectivity, paired test was chosen.

Tests for statistical significance were performed separately on each of the 3 parameters to obtain group-level ROI-specific differences in HRF parameters. To be conservative, an intersection of the differences in the 3 parameters was taken to finally obtain a $58 \times 58$ binary matrix of significant HRF differences. This was then combined with significant connectivity differences obtained in the previous step (i.e., found a common matrix of significance by overlapping the 2) to obtain the following final differences of interest: (1) pseudo-negatives (true effects potentially corrupted and diminished by HRF variability): DC $>$ NDC overlapped with (intersection) HRF differences, and (2) pseudo-positives (potentially untrue effects arising because of HRF variability): NDC $>$ DC overlapped with HRF differences. These 2 observations enabled us to test our hypothesis.

For the significant connections identified with the above procedure, as well as for the entire data, we probe the magnitude of change observed in FC and the corresponding change in HRF parameters. We present and discuss the summary statistics and range of values taken by the percentage FC difference between NDC and DC ( $\triangle \mathrm{FC}$ as in Eq. (5)) as well as the difference in $\mathrm{HRF}$ parameters $(\Delta \mathrm{RH}, \Delta \mathrm{TTP}$, and $\triangle \mathrm{FWHM}$ as in Eqs. (6-8)).

\section{7 | Follow-up HRF analysis}

As a follow-up analysis, we probed deeper into the magnitude of difference in connectivity between deconvolved and non-deconvolved data. The goal was to derive quantitative relationships between the amount of HRF variability and the amount of its impact on connectivity modeling, which could be useful for guiding future fMRI FC studies.

Specifically, we derived the relationship between experimental $\triangle F C$ and the percentage of connections $(P C)$ with at least the corresponding $\triangle \mathrm{FC}$. To perform this computation, we considered all ROIs and all connections without any exclusions. Given that we had 58 ROIs and 47 subjects, our $58 \times 58 \times 47 \Delta \mathrm{FC}$ matrix contained $Q=155,382$ connections/paths excluding the diagonal elements. $P C(n)$ was computed as the percentage of these $Q$ connections/paths that had a $\Delta \mathrm{FC} \geq n$.

$$
P C(n)=\frac{100}{Q} \sum_{i=1}^{Q} \sum_{\Delta F C \geq n} 1,
$$

where $i$ corresponds to the $i^{\text {th }}$ among $Q$ connections/paths. In summary, $\triangle F C$ was an estimated measure of the impact of HRF variability on FC (as in Eq. (5)), whereas PC was a measure of the percentage of all connections affected by that magnitude of HRF variability (or more). Examples from findings in the Results section would illustrate these concepts lucidly.

\section{3 | RESULTS}

\section{1 | Simulation results}

We performed simulations to empirically assess the percentage change in FC $(\Delta F C)$ caused by the difference in HRF parameters between the corresponding 2 regions (i.e., $\Delta \mathrm{RH}$, $\triangle T T P$, and $\triangle F W H M$ ) across a range of biologically plausible values of these measures. Here, we present the graphs for $\Delta \mathrm{FC}$ versus $\Delta \mathrm{RH}$ (Figure $3 \mathrm{~A}$ ), $\Delta \mathrm{FC}$ versus $\Delta \mathrm{TTP}$ (Figure $3 \mathrm{~B}$ ), $\Delta \mathrm{FC}$ versus $\triangle \mathrm{FWHM}$ (Figure $3 \mathrm{C}$ ), and $\Delta \mathrm{FC}$ versus absolute neural-FC (Figure 3D). Because we used experimental data with $\mathrm{TR}=1$, plots in Figure 3 have been computed with $\mathrm{TR}=1$ (see Supporting Information Figure S1 for similar plots across all TRs). $\Delta \mathrm{FC}$ and $\Delta \mathrm{RH}$ correspond to the percentage change in them, whereas $\Delta \mathrm{TTP}$ and $\triangle F W H M$ are in seconds. With the case of identical HRFs $(\Delta \mathrm{RH}=\Delta \mathrm{TTP}=\Delta \mathrm{FWHM}=0), \Delta \mathrm{FC}$ was insignificant $\left(P=0.9999\right.$, mean $\left.\pm \mathrm{SD}=10^{-15} \pm 0.006\right)$, which validates our deconvolution approach in this regard. Within the acceptable physiological range of variation in the HRF parameters 


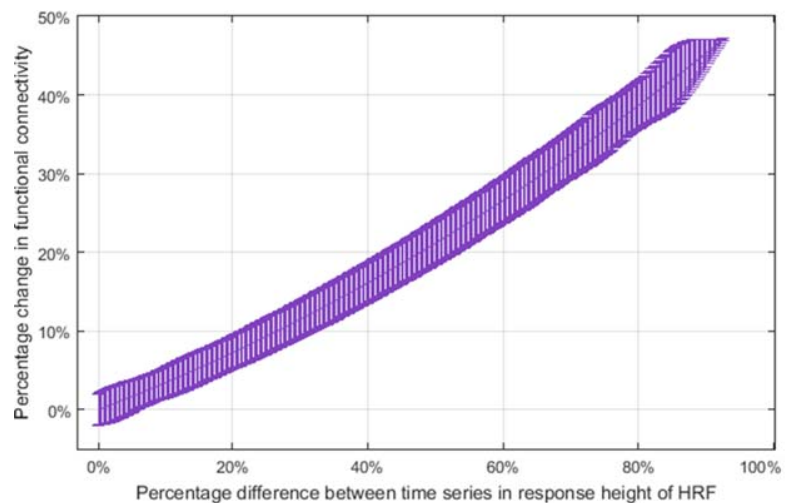

(A)

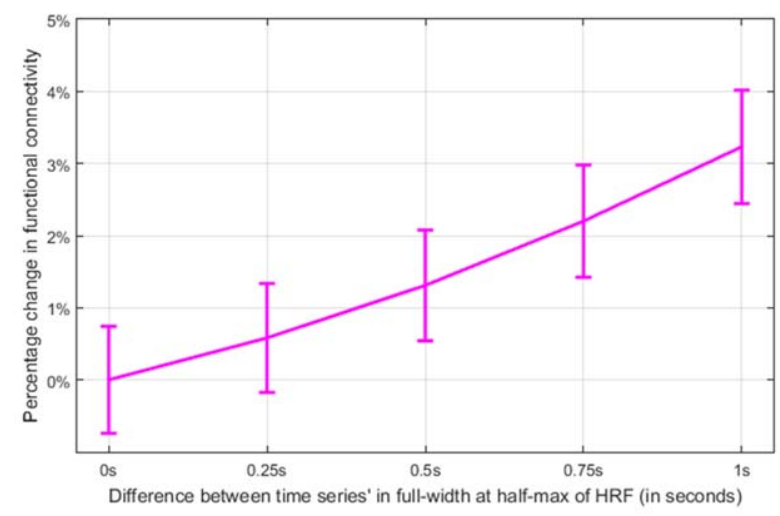

(C)

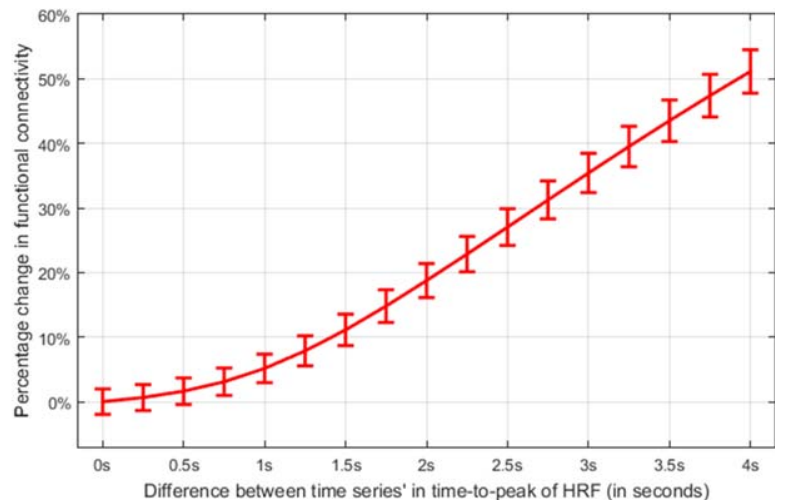

(B)

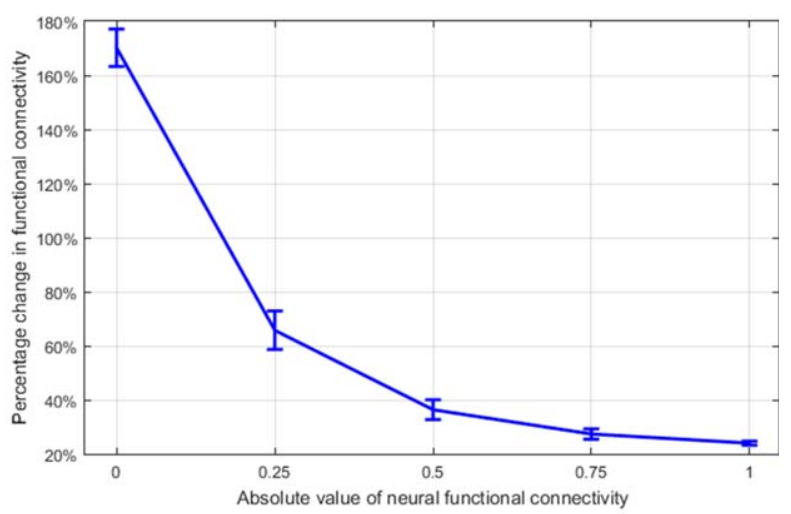

(D)

F I G URE 3 Simulation results for empirical assessment of percentage change in functional connectivity $(\triangle \mathrm{FC})$ between neural and BOLD time series caused by difference in HRF parameters ( $\triangle \mathrm{RH}, \Delta \mathrm{TTP}, \Delta \mathrm{FWHM}$ ) between the corresponding 2 time series, across all possible physiologically plausible values of these HRF measures. (A) $\Delta \mathrm{FC}$ versus $\Delta \mathrm{RH}$ ( $\mathrm{RH}$ resolution $=0.25 \%$ ). (B) $\Delta \mathrm{FC}$ versus $\Delta \mathrm{TTP}$ (TTP resolution $=0.25 \mathrm{~s}$ ). (C) $\Delta \mathrm{FC}$ versus $\Delta$ FWHM (FWHM resolution $=0.25 \mathrm{~s})$. (D) $\Delta \mathrm{FC}$ versus absolute value of neural $\mathrm{FC}(\mathrm{FC}$ resolution $=0.25)$. Error bars show $1 \mathrm{SD}$ above and below the mean. The physiologically plausible lower and upper bounds of HRF parameters were obtained from Handwerker et al. ${ }^{2}$ We observed robust and nearly linear-positive relationship between $\triangle \mathrm{FC}$ and change in HRF parameters. Up to $50 \%$ change in FC was observable because of either RH or TTP variability. Smaller FC values were more vulnerable to HRF variability. RH, response height; TTP, time-to-peak; FWHM, full-width at half-max; FC, functional connectivity

presented here, ${ }^{2}$ simulations showed a nearly linear positive relationship between changes in FC and HRF parameters, with RH and TTP causing up to 50\% change in FC. FWHM had a smaller impact of up to $5 \%$. Additionally, the largest impact on FC was for weaker connection strengths (up to $170 \%$ change), and the impact reduced to as low as $24 \%$ for the strongest connections (that is still a considerable error). We found that $\triangle F C$ was not significantly different across TRs for specific alterations of HRF parameters, except with FWHM at $1 \mathrm{~s}$ (Supporting Information Figure S1C). However, when $\triangle F C$ across all possible alterations of HRF parameters were considered, there was significant increase in $\Delta \mathrm{FC}$ with TR $\left(P=10^{-36}, F=56.61\right)$, with $\Delta \mathrm{FC}=36.1 \pm$ 8.9 for $\mathrm{TR}=0.5$ and $\Delta \mathrm{FC}=40.2 \pm 9.8$ for $\mathrm{TR}=2$, implying that poorer temporal resolution worsens the impact of HRF variability on FC estimates (see the Supporting Information [section S2] for the effect of TR on FC-HRF relationship). In summary, simulations revealed the landscape and limits of the magnitude of the impact of HRF variability on our ability to estimate FC accurately at the neural level.
The deconvolution technique's original paper ${ }^{21}$ has presented several simulations illustrating the validity of the technique. Here, we provide 2 sanity checks for providing further validation of the deconvolution procedure. First, a fundamental claim of deconvolution is if a pair of time series have identical HRFs, then their correlation would be the same with or without deconvolution, and the deconvolution approach should be able to re-identify the correlation between the underlying neural event time series. Second, if 2 time series have different HRFs, then the deconvolution approach should be able to accurately access the true neural correlation, but analysis without deconvolution would not. Using simple simulations, we demonstrate the construct validity of the deconvolution technique based on the above principles. Further details can be obtained from Supporting Information S1.

\section{$3.2 \mid$ HRF results}

Figure 4 shows the 58 DMN ROIs used in this work (please refer to Supporting Information Table S1 for the MNI 

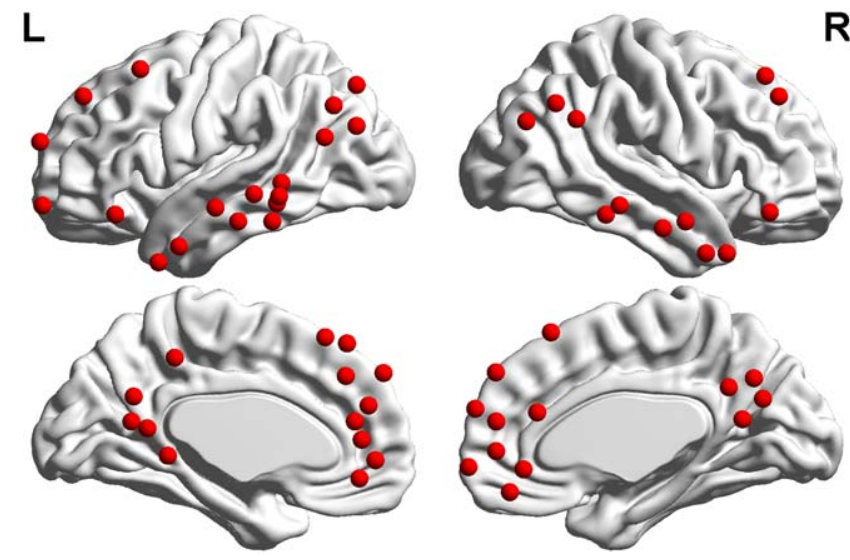

FIG URE 4 ROIs of the default mode network provided by Power et al. ${ }^{20}$ used in this work (see Supporting Information Table S2 for details of the template)

coordinates and names of these ROIs). Figures $5 \mathrm{~A}$ and $5 \mathrm{~B}$ show the pseudo-negative connections potentially arising from HRF variability. They were obtained as those connections that had lower connectivity with the HRF-variability-ignored traditional fMRI data compared to the HRF-variability-reduced deconvolved data, along with differences in HRF between the associated ROIs (detailed statistics available in Supporting Information Table S2). Figures 5C and 5D show the pseudopositive connections, which exhibited higher connectivity in HRF-variability-ignored data, along with HRF differences between associated ROIs (detailed statistics available in Supporting Information Table S3). Medial prefrontal, anterior cingulate, and temporal pole showed qualitatively drastic reduction in connectivity after deconvolution. We repeated the analysis using spatially non-smoothed fMRI data, and did not find any significant differences in the HRF maps between the smoothed and non-smoothed data (for details of the analysis and discussion of corresponding results with non-smoothed data, please refer to the Supporting Information [section S4]). Further, we investigated whether deconvolution could introduce higher frequencies into the deconvolved signal and whether such spectral differences could drive diverging results from DC and NDC data. However, we found no significant difference between the spectral characteristics of the raw BOLD data and deconvolved data (for details of the analysis and discussion of corresponding results, please refer to the Supporting Information [section S5]).

It must be noted that we performed a paired t-test to test for statistical significance, because we were comparing connectivities of the same individuals with different preprocessing pipelines. We identified a large number of pseudo-connectivities even with a conservative family-wiseerror-corrected (Bonferroni) threshold. Instead, using a 2sample t-test here (although inappropriate to use in our context), resulted in far less number of significant connections (just 4 connections at $P<0.05$, uncorrected). This observation deserves further attention.
As an example, Figure 6 shows the connectivity values for all 47 participants with both pipelines (deconvolved and non-deconvolved), for 1 of the identified pseudo-positive connectivity paths (between precuneus and superior frontal gyrus, corresponding to regions 13 and 46 in Supporting Information Table $\mathrm{S} 1$ ), whose regions had significantly different RH $\left(P=2 \times 10^{-20}\right)$, TTP $\left(P=10^{-88}\right)$, and FWHM $\left(P=10^{-80}\right)$. What we observed explains the reason for the large disparity in the results between the use of a paired t-test and a 2-sample t-test. At the individual subject level, we observed that there was a consistent shift in connectivity value in the same direction, and this trend was highly reproducible for all participants, with NDC values consistently being higher than $\mathrm{DC}$ values $(\Delta \mathrm{FC}=15.34 \pm 4.85 \%)$. For this reason, the paired t-test returned high statistical significance for this path. However, the variability across participants was notably larger $\left(\mathrm{SD}_{\mathrm{NDC}}=0.16, \mathrm{SD}_{\mathrm{DC}}=0.15\right)$ than the variability within participants caused by HRF differences (pairwise difference in $\mathrm{FC}=0.06 \pm 0.02$ ), which means that the group mean values might not be significantly different even if considerable differences were observed at the individual-subject level. This explains why the 2-sample ttest returned far less number of significant connectivities. For this reason, we also did not observe any notable differences in, say, the default-mode's functional network structure between deconvolved and non-deconvolved data when using a 2 sample t-test, because individual-level differences because of HRF variability are often buried under larger inter-subject variability.

Next, we probed deeper into the magnitude of change observed in $\mathrm{FC}$ after deconvolution $(\Delta \mathrm{FC})$, as well as the summary statistics of the HRF parameters obtained from deconvolution. These numbers were obtained for 3 cases: (1) Entire data: all the ROIs and connections, without exclusions; (2) Same as previous case but with outliers removed. Because the histogram of all these measures resembled a decaying exponential with a long tail (more about this later), we discarded outliers to provide a more balanced view of their central tendencies. Outliers were determined as values that exceeded 3 scaled median absolute deviations away from the median. (3) Only for those connections and associated ROIs that exhibited significant difference between NDC and DC (plus outliers removed); which was performed to compare values from significant connections against the entire connectome obtained in case (1).

Table 1 provides the summary statistics of various measures for all 3 cases. We observed lower FC after deconvolution compared to before deconvolution (corrected $P<0.05$ ) with the entire data as well as significant connections that corroborates with the observation of more pseudo-positives than pseudo-negatives in the connectivity results. We found $30.5 \%$ mean error and $11.5 \%$ median error in FC caused by HRF variability with the entire data. After discarding outliers (12.9\% of the connections), the mean and median $\triangle \mathrm{FC}$ were 


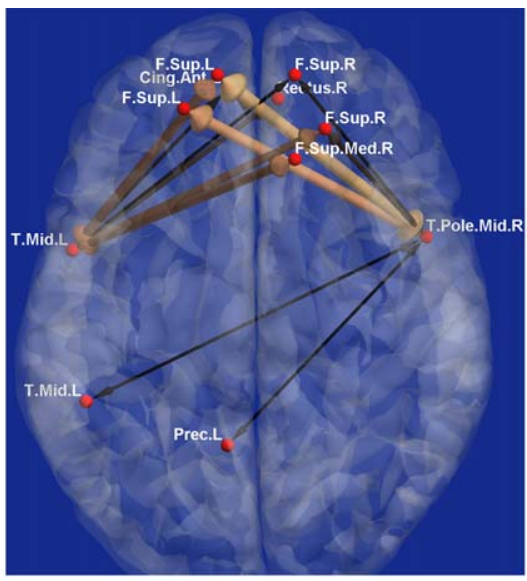

(A)

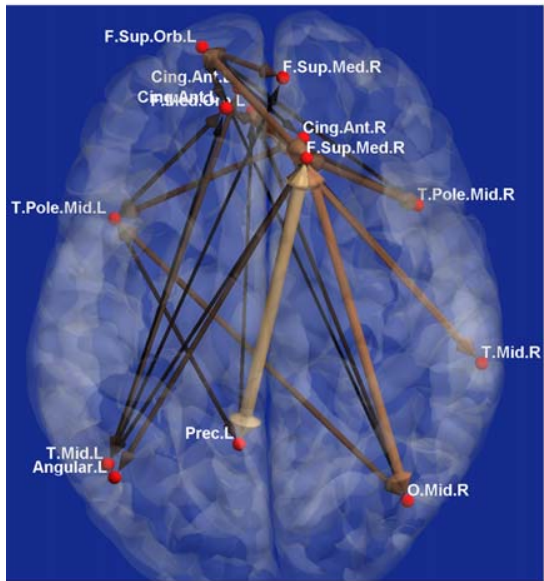

(C)

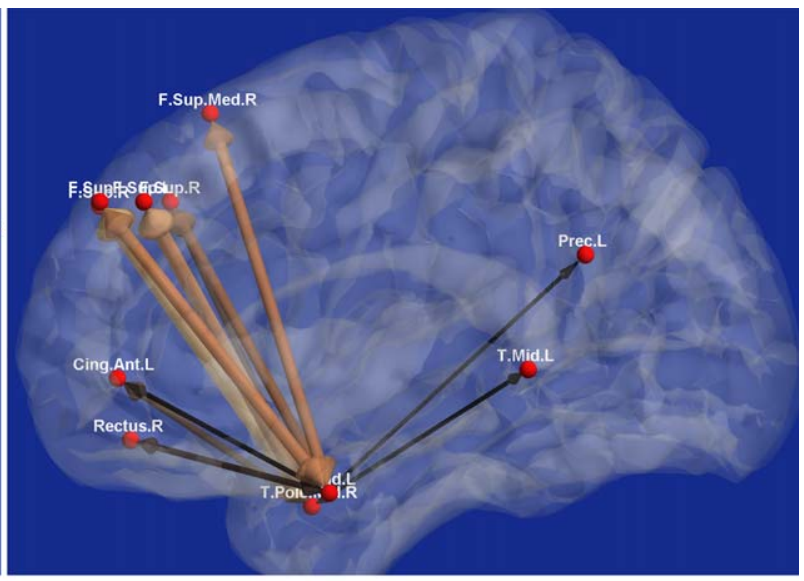

(B)

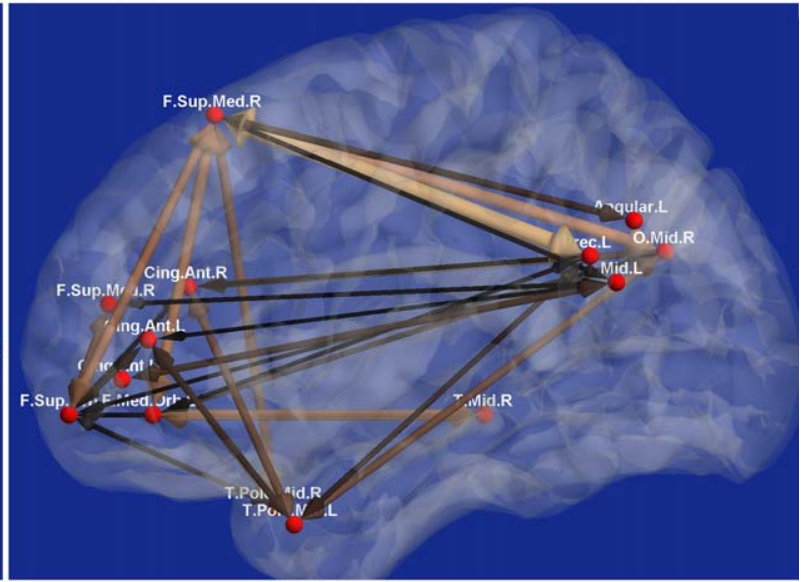

(D)

F IG URE 5 Pseudo-positive and pseudo-negative connections in the DMN arising from HRF variability. Thicker connections and lighter color corresponds to higher $T$-value. Please refer to Supporting Information Table S1 for expansion of abbreviations and information on the ROIs. Pseudo-negatives: (A) axial view; (B) sagittal view. Pseudo-positives: (C) axial view; (D) sagittal view

$14.7 \%$ and $9.6 \%$ respectively, which was substantial considering that the effect sizes we often observe in cognitive, neurologic, and psychiatric neurosciences are of similar or smaller magnitude. ${ }^{34}$ Although smaller than the former case, especially the mean, it must be noted that these outliers (12.9\% in our data), show large percentage change in $\mathrm{FC}$, exist in any data, and impact any FC analysis performed using it. It should also be noted that outliers may sometimes be the connections of interest because network-level disruptions because of pathology or cognitive manipulations are often seen in a small percentage of all possible connections. Still, to be conservative, in this study, we focused on the results obtained after eliminating outliers (henceforth we do not mention about outlier removal, but it is implied).

Studying the distribution of $\triangle \mathrm{FC}$ would provide further insights not available through summary statistics, especially because it is non-Gaussian. Figure 7 provides the histogram of $\triangle \mathrm{FC}$ obtained from entire data, along with the mean, median, and $95 \%$ interval after outlier removal. The distribution resembles a decaying exponential, with the mean of $14.7 \%$ being a reasonable estimate of the overall error in FC
( $\triangle \mathrm{FC}$ ) caused by HRF variability. In the specific example considered in Figure 6, the mean $\triangle \mathrm{FC}$ was $15.34 \%$.

Referring to Table 1, when considering only the significant connections (as in Figure 5), $\triangle \mathrm{FC}$ was significantly larger than the case of entire data $\left(P=10^{-22}\right)$, with the mean and median $\triangle \mathrm{FC}$ being $17.1 \%$ and $11.2 \%$, respectively. This was expected, given that significant connections were, by definition, different between DC and NDC. However, the relatively close error of $14.7 \%$ with the entire data (that was $14.6 \%$ if significant connections were excluded from entire data) meant that HRF variability had a widespread impact even among those connections that were not significantly different between DC and NDC (with our strict statistical threshold).

Understandably, the difference in FC because of HRF variability was more than the difference in $\mathrm{RH}$, and $\mathrm{FC}$ was also more variable and had more outliers, given that $\mathrm{FC}$ is impacted by all $3 \mathrm{HRF}$ parameters. Interestingly, $\triangle \mathrm{FC}$ was larger by $17 \%$ with significant connections (compared to entire data), $\Delta$ TTP by $16 \%$, and $\triangle \mathrm{FWHM}$ by $17 \%$, whereas $\Delta \mathrm{RH}$ was not different. This curious observation hints that 


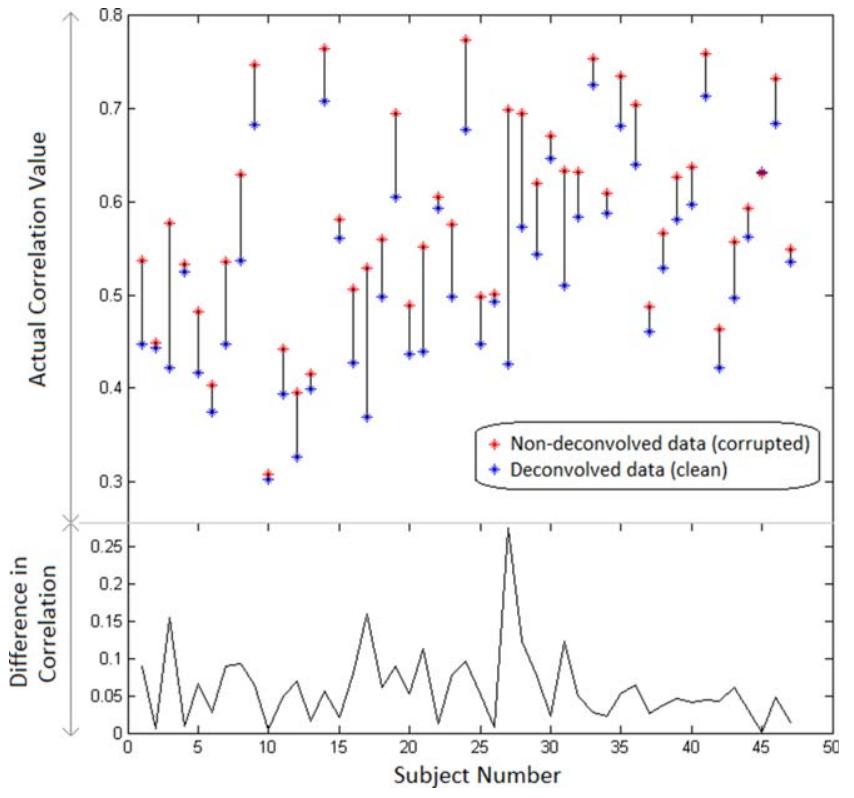

F I G URE 6 Individual connectivity estimates of a connectivity path (the pseudo-positive connection with highest $T$-value taken as an example here). Connectivities are shown for all 47 subjects, obtained with both deconvolved (DC, HRF-variability-reduced) and non-deconvolved (NDC, HRF-variability-ignored) data. Black lines are drawn connecting the DC and NDC values in every subject, illustrating what the paired t-test captures. These differences show the magnitude of change caused by HRF variability in each subject, which is the effect of interest in this work. The same differences are plotted at the bottom part of the figure. In this example, we observe that HRF-variability-ignored NDC data displays a shift from DC's true group-average connectivity value by 0.06 . However, because the connectivity values are themselves scattered across subjects with large variance, a 2-sample t-test between DC and NDC values does not result in high statistical significance

TTP and FWHM, which causes timing errors in the data as seen in Figure 2, contributed to a greater extent toward significant changes in FC than toward non-significant connections, whereas RH had a uniform impact on significant and non-significant connections, hinting that variability in TTP/ FWHM might disproportionately affect highly impacted connections.

Mapping these numbers to the simulation results (as in Figure 3), the mean $\pm \mathrm{SD}$ of experimental $\Delta \mathrm{RH}$ values (as in Table 1) corresponded to $\Delta \mathrm{FC}$ of $6.39 \pm 1.87 \%$ (Figure 3A), $\Delta \mathrm{TTP}$ to $\Delta \mathrm{FC}$ of $6.37 \pm 2.37 \%$ (Figure $3 \mathrm{~B}$ ), and $\Delta \mathrm{FWHM}$ to $\Delta \mathrm{FC}$ of $2.27 \pm 0.48 \%$ (Figure $3 \mathrm{C}$ ). The mean $\Delta \mathrm{FC}$ in experimental data $(=14.7 \%)$ was close to the sum of mean values noted above from the simulated $\Delta \mathrm{FCs}(=15 \%)$. We do not imply that changes RH, TTP, and FWHM share a linear additive relationship with $\triangle \mathrm{FC}$. We could not test this in the simulation because it does not permit simultaneous control over all 3 HRF parameters ${ }^{15}$ (because RH, TTP, and FWHM are biophysically related), ${ }^{35}$ and this is an aspect that may be investigated in the future.
These findings imply that the combined effect of HRF parameters on FC in real data is at least as bad as the simulation results, and HRF variability, on average, causes $\sim 14.7 \%$ error in the FC data in healthy controls for the scan parameters we have used in a 7T scanner. The HRF parameters as well as connectivity data have been made publicly avail$\mathrm{able}^{33}$ to help researchers replicate these findings.

\section{3 | Follow-up HRF results}

As a follow-up analysis, we derived quantitative relationships between the amount of HRF variability and the amount of its impact on FC modeling, which could have practical use in guiding future fMRI FC studies regarding the impact of HRF variability. Figure 8A depicts the relationship between the percentage of connections $(P C)$ affected by HRF variability and the percent connectivity difference $(\triangle \mathrm{FC})$ between deconvolved and non-deconvolved data. A double exponential curve could fit this $P C$ versus $\triangle \mathrm{FC}$ graph with $R^{2}=0.9998$, which is given by the following equation:

$y(x)=0.75 e^{-0.19 x}+0.39 e^{-0.04 x} ;$ where $x=\Delta \mathrm{FC}$ and $y=P C$.

It is notable that these findings were obtained from the entire connectivity data, including outliers, without limiting to only the identified significant connectivities. This graph shows that, for example, there was at least $50 \%$ difference in the magnitude of connectivity between NDC and DC in $6.5 \%$ of the connections, there was at least $25 \%$ difference in the magnitude of connectivity between NDC and DC in $16.2 \%$ of the connections, and at least $10 \%$ difference between NDC and DC in $38.5 \%$ of the connections. Also, $50 \%$ of all the connections had at least $7 \%$ connectivity difference between NDC and DC, and 10\% of all the connections had as much as $37 \%$ difference in connectivity. These findings provide deeper insights into what percentage of connections are impacted by HRF variability and by what magnitude.

Given that deconvolution is an estimation procedure (like most other imaging-based methods), questions could arise about the quality of the HRF estimation, although the deconvolution procedure used by us has been widely accepted and extensively used. ${ }^{5,22-29}$ For the sake of argument, however, even if we were to say that our estimated HRFs post deconvolution were largely inaccurate, it is still undeniable that, theoretically, the ground-truth HRF varies considerably across the brain, across individuals, and across disease groups. ${ }^{2,3}$ Our findings, in the worst case, at least illustrate how HRF variability can result in widespread confounds in FC estimates. To illustrate this point, we picked $25 \%$ of all the connections that exhibited the least difference in the HRF parameters between the corresponding regions and 
TA B LE 1 Summary statistics of various measures for 3 cases

\begin{tabular}{|c|c|c|c|c|c|}
\hline Measure & Mean \pm SD & $\begin{array}{l}\text { Median } \pm \text { absolute } \\
\text { deviation }\end{array}$ & $\mathbf{9 5 \%}$ interval & [min, $\max ]$ & $\%$ eliminated \\
\hline \multicolumn{6}{|c|}{ (A) Entire data without exclusions } \\
\hline $\mathrm{FC}^{\mathrm{NDC}}$ & $0.302 \pm 0.112$ & $0.258 \pm 0.149$ & {$[0.012,0.847]$} & {$[0,0.97]$} & - \\
\hline $\mathrm{FC}^{\mathrm{DC}}$ & $0.294 \pm 0.110$ & $0.249 \pm 0.146$ & {$[0.012,0.839]$} & {$[0,0.96]$} & - \\
\hline$\left[\mathrm{FC}^{\mathrm{NDC}}-\mathrm{FC}^{\mathrm{DC}}\right]$ & $0.036 \pm 0.016$ & $0.028 \pm 0.018$ & {$[0.001,0.117]$} & {$[0,0.29]$} & - \\
\hline$\Delta \mathrm{FC}(\%)$ & $30.54 \pm 14.21$ & $11.51 \pm 9.10$ & {$[0.13,207.04]$} & {$[0,499.37]$} & - \\
\hline$\Delta \mathrm{RH}(\%)$ & $17.71 \pm 4.75$ & $13.03 \pm 7.51$ & {$[0.21,72.29]$} & {$[0,166.63]$} & - \\
\hline$\Delta \mathrm{TTP}(\mathrm{s})$ & $0.944 \pm 0.28$ & $0.566 \pm 0.43$ & {$[0.006,5.384]$} & {$[0,6.42]$} & - \\
\hline$\Delta \mathrm{FWHM}(\mathrm{s})$ & $0.948 \pm 0.28$ & $0.575 \pm 0.43$ & {$[0.007,5.381]$} & {$[0,6.51]$} & - \\
\hline \multicolumn{6}{|c|}{ (B) Entire data with outliers eliminated ${ }^{\mathrm{a}}$} \\
\hline$\Delta \mathrm{FC}(\%)$ & $14.68 \pm 3.59$ & $9.59 \pm 7.01$ & {$[0.35,53.99]$} & {$[0,62.95]$} & 12.90 \\
\hline$\Delta \mathrm{RH}(\%)$ & $14.15 \pm 2.61$ & $12.32 \pm 6.69$ & {$[0.61,40.69]$} & {$[0,48.55]$} & 6.27 \\
\hline$\Delta \mathrm{TTP}(\mathrm{s})$ & $0.793 \pm 0.17$ & $0.553 \pm 0.41$ & {$[0.019,2.272]$} & {$[0,2.83]$} & 3.46 \\
\hline$\Delta \mathrm{FWHM}(\mathrm{s})$ & $0.797 \pm 0.17$ & $0.561 \pm 0.41$ & {$[0.021,2.288]$} & {$[0,2.87]$} & 3.45 \\
\hline \multicolumn{6}{|c|}{ (C) Significant connections/ROIs ${ }^{\mathrm{b}}$} \\
\hline $\mathrm{FC}^{\mathrm{NDC}}$ & $0.356 \pm 0.122$ & $0.312 \pm 0.178$ & {$[0.017,0.880]$} & {$[0,0.97]$} & 96.79 \\
\hline $\mathrm{FC}^{\mathrm{DC}}$ & $0.333 \pm 0.118$ & $0.287 \pm 0.163$ & {$[0.014,0.870]$} & {$[0,0.96]$} & 96.79 \\
\hline$\left[\mathrm{FC}^{\mathrm{NDC}}-\mathrm{FC}^{\mathrm{DC}}\right]$ & $0.046 \pm 0.019$ & $0.036 \pm 0.024$ & {$[0.002,0.142]$} & {$[0,0.27]$} & 96.79 \\
\hline$\Delta \mathrm{FC}(\%)$ & $17.11 \pm 4.25$ & $11.21 \pm 8.62$ & {$[0.39,62.55]$} & {$[0,74.90]$} & 11.40 \\
\hline$\Delta \mathrm{RH}(\%)$ & $14.09 \pm 2.59$ & $12.47 \pm 6.84$ & {$[0.52,41.83]$} & {$[0,48.69]$} & 6.64 \\
\hline$\Delta \mathrm{TTP}(\mathrm{s})$ & $0.918 \pm 0.15$ & $0.879 \pm 0.55$ & {$[0.035,2.072]$} & {$[0,3.55]$} & 0.00 \\
\hline$\Delta \mathrm{FWHM}(\mathrm{s})$ & $0.929 \pm 0.15$ & $0.889 \pm 0.54$ & {$[0.031,2.099]$} & {$[0,3.48]$} & 0.00 \\
\hline
\end{tabular}

Abbreviations: ROIs, regions of interest.

The measures were functional connectivity (FC) from non-deconvolved data $\left(\mathrm{FC}^{\mathrm{NDC}}\right)$, $\mathrm{FC}$ from deconvolved data $\left(\mathrm{FC}^{\mathrm{DC}}\right)$, percentage change in $\mathrm{FC}$ as defined in Eqn. $5(\Delta \mathrm{FC})$, percentage change in response height as defined in Eqn. $6(\Delta \mathrm{RH})$, change in time-to-peak as defined in Eqn. $7(\Delta \mathrm{TTP})$, and change in full-width at half-maximum as defined in Eqn. $8(\Delta \mathrm{FWHM})$. The column " $\%$ eliminated" shows the percentage of outliers eliminated in $\Delta \mathrm{FC}, \Delta \mathrm{RH}, \Delta \mathrm{TTP}$, and $\Delta \mathrm{FWHM}$, and includes the percentage of connections found to be not significant in $\mathrm{FC}^{\mathrm{NDC}}$ and $\mathrm{FC}^{\mathrm{DC}}$ in $(\mathrm{C})$.

a Defined as values exceeding 3 scaled absolute deviations above the median.

${ }^{\mathrm{b}}$ Only significant connections and their associated ROIs (outliers plus non-significant connections and associated ROIs eliminated).

performed similar analysis as before to compare $\triangle \mathrm{FC}$ and $P C$ only for these $25 \%$ of the connections. This represents a best-case scenario for FC, wherein the HRF variability is low. Even in such a scenario, we found that connectivities derived from deconvolved and non-deconvolved data differed considerably (Figure $8 \mathrm{~B}$ ). The $P C-\Delta \mathrm{FC}$ relationship was expectedly better; still, we found that there was at least $50 \%$ difference in connectivity magnitude between NDC and DC in $5.8 \%$ of the connections, there was at least $25 \%$ difference between NDC and DC connectivities in $14.6 \%$ of the connections, and at least $10 \%$ difference between NDC and DC connectivities in $35.7 \%$ of the connections. Ten percent of all the connections had as much as 34\% difference in connectivity. As we can see, even if one were to contend that the ground-truth HRFs were less variable or if our deconvolution estimates were considerably noisy, one would still observe the prominent effect of HRF variability on FC. As such, ignoring HRF variability in fMRI FC analysis would invariably cause considerable error in $\mathrm{FC}$ estimates.

\section{4 | DISCUSSION}

In this work, we tested the hypothesis that FC in the DMN is affected by HRF variability, which is (at least in part) nonneural in origin and hence undesirable. We provided the theoretical background on which this hypothesis is based, followed by simulations to provide empirical evidence to back our hypothesis when the ground truth is known. Further, we found experimental evidence to support our hypothesis. We found functional connections that were significantly different in the same participants between HRF-variability-ignored (NDC) and HRF-variability-reduced (DC) data. Additionally, HRF parameters were found to be significantly different between the regions involved in all such connections, therefore attributing the connectivity differences to HRF variability. These findings have considerable implications for the interpretability and reliability of FC findings in fMRI studies. Results imply that HRF variability could lead to the identification of false positive and false negative connections, and resting-state fMRI FC studies that do not account for this 


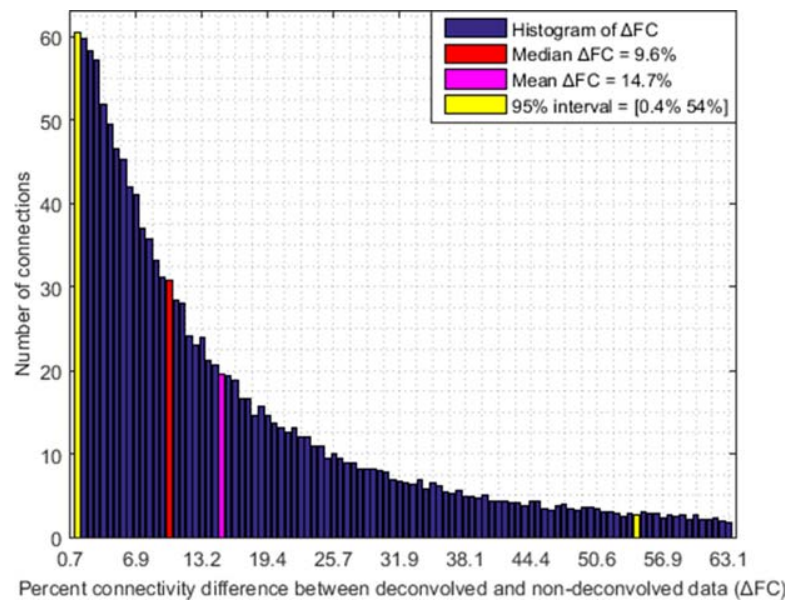

F I G URE 7 The histogram of percentage connectivity error $(\Delta \mathrm{FC})$, obtained from the entire connectivity data (after excluding outliers). Specifically, it shows the plot of the number of connections corresponding to the various range of values of percentage connectivity difference between deconvolved (DC) and non-deconvolved (NDC) data ( $\triangle \mathrm{FC}$ ), as defined in Eq. 5. Because our data had 58 ROIs, there were a total of 1653 connections. Outliers, defined as values exceeding 3 scaled median absolute deviations above the median, were excluded, which brought the range of $\Delta \mathrm{FC}$ to $[0,63 \%]$. Note that the distribution of $\Delta \mathrm{FC}$ would have had a much longer tail if outliers were included. The histogram was divided into 100 bins, hence the value at each bin (no. of connections) corresponded to a range of $0.63 \%$. The red bar shows the median connectivity error of $9.6 \%$ caused by HRF variability, whereas the magenta bar shows the mean of $14.7 \%$. Yellow bars show the $95 \%$ interval $[0.4,54]$ percent

variability would need to interpret their findings with caution.

Simulations results (Figure 3 ) showed a nearly linear positive relationship between $\triangle \mathrm{FC}$ and difference in HRF parameters. RH and TTP could independently cause a change of up to $50 \%$ in FC, whereas FWHM could cause about 5\% change. Simulations provide the limits and formal relationships between the entities being studied here and help us better understand the experimental results as well as the possibilities not evident from our experimental data. It is clear that HRF variability, if ignored, is a considerable confound in FC analysis.

Looking into the pseudo-negative and pseudo-positive connections identified in this work (Figure 5), we found that most of the affected connections were between different lobes of the brain. Pseudo-negatives (Figures 5A and 5B) were predominantly observed in connections between medial frontal and middle temporal regions. In fact, none of the altered connection were within the same lobe. Pseudopositives (Figures 5C and 5D) were predominantly observed in connections between parietal and medial frontal regions, whereas some connections between those regions and middle temporal regions were also observed. Taken together, this trend suggests that connections between functionally distinct and anatomically distant regions are more susceptible to corruption by HRF variability. This could be attributed to the fact that the neurochemistry and vasculature between such distant and distinct regions differ by a larger extent, ${ }^{2,3}$ leading to larger differences in HRF (that depends on cerebrovascular reactivity and neurovascular coupling), ${ }^{1}$ and therefore larger impact on connectivity.

To better understand this pattern of inter-lobe connectivities being maximally impacted by HRF variability, we discuss the underlying neurochemistry in further detail. It is known that neuromodulators released by GABAergic and glutamatergic interneurons directly modulate local cerebral blood flow, ${ }^{36}$ and hence the HRF. ${ }^{37}$ Lower gamma-aminobutyric-acid (GABA) concentration results in quicker, taller, and narrower HRF. ${ }^{38}$ Glutamate acts on N-methyl-Daspartate (NMDA) receptors causing dilation of blood vessels, ${ }^{39}$ hence influencing the HRF. Serotonin, a vasoconstrictor that provides blood-brain barrier permeability, modulates neurovascular coupling, and hence the HRF. ${ }^{40}$ These neurochemical processes are known to vary across the brain, with larger differences being more likely between distinct and distant regions. ${ }^{2,3}$ Vasculature is also inconsistent across the brain, hence the HRF would be different between brain regions neighboring larger blood vessels compared to smaller ones. ${ }^{3}$ However, it is to be noted that this is a simplistic explanation of much complex underlying neurochemical and neurovascular phenomena. Therefore, for a more accurate and complete picture, we refer the readers to Hillman. ${ }^{41}$ It is also noteworthy that a recent study by Murphy et al. ${ }^{42}$ demonstrated that resting-state connectivity obtained from fMRI and that from calcium imaging significantly differ in many brain regions, and they hypothesize that such differences could in-part be explained by the regional variability of the HRF. Our results provide credence to this view.

Another interesting observation was that the number of pseudo-positives were more than the number of pseudonegatives by a substantial margin. Although we reported only the results obtained with a conservative statistical threshold $(P<0.05$, Bonferroni corrected), we observed that this trend holds true even with other more liberal statistical thresholds (tested up to $P<0.05$ uncorrected). In brain imaging, pseudo-positives are more undesirable than pseudonegatives, because, in most cases, reporting a connection that does not exist is more detrimental to the conclusions than a true connection that was not identified. Hence, more caution must be exercised, as HRF variability predominantly seems to cause more pseudo-positives.

We observed that the impact of HRF variability on FC often gets buried in the larger inter-subject variability of connectivity (Figure 6). However, this must not be misunderstood to mean that HRF variability is not a concern, especially after observing the results for percentage error in connectivity $(\Delta \mathrm{FC})$. HRF variability could have detrimental effects when comparing participants from different groups, 


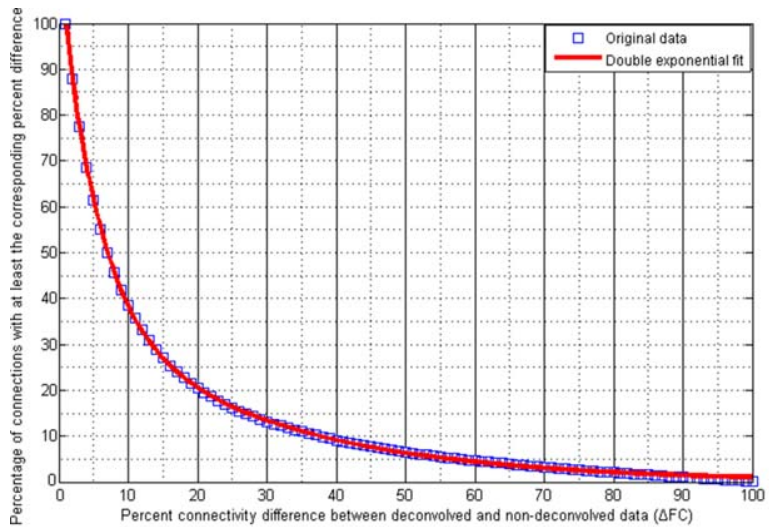

(A)

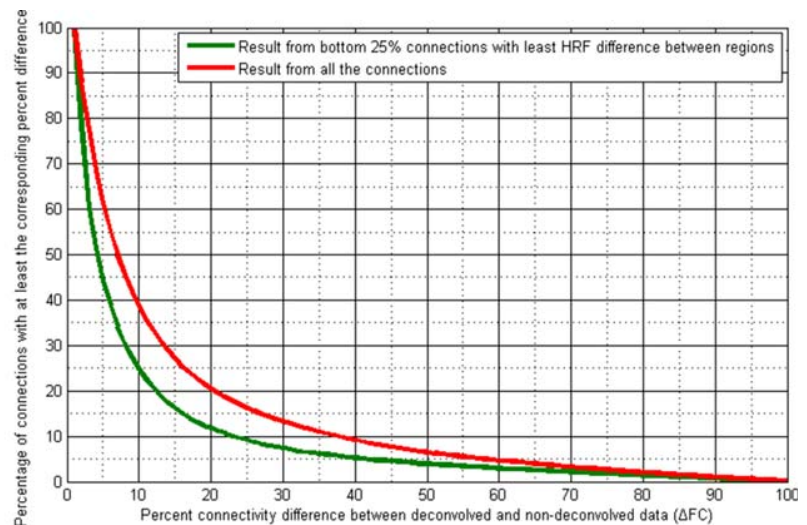

(B)

FIG URE 8 Quantitative relationship between the amount of HRF variability and the amount of its impact on functional connectivity modeling. (A) Relationship between the percentage of connections $(P C)$ affected by HRF variability and the percent connectivity difference $(\Delta \mathrm{FC})$ between deconvolved and non-deconvolved data; shows original data as well as double exponential curve fit $\left(R^{2}=0.9998\right)$. The graph shows, for example, that there was at least $10 \%$ connectivity difference in one-third of all connections, and $10 \%$ of all connections had as much as one-third difference in connectivity value. (B) The same curve fit as in the previous case (in red), along with the curve fit obtained by only $25 \%$ of the connections that exhibited least difference in HRF (in purple). This represents a conservative scenario in which the HRF variability is less, yet considerably large number of connections were impacted. Notably this was obtained from entire dataset, not just from significant differences

given that group-level connectivity differences are often of similar magnitude or even smaller than the error $\triangle \mathrm{FC}$ reported here. ${ }^{34}$ Across the entire data, the average percentage error caused in FC by HRF variability was $30.5 \%$, with error being $14.7 \%$ after outlier elimination. Such large impact was also observable in the example in Figure 6 (mean $\Delta \mathrm{FC}$ was $15.3 \%$ ). The distribution of $\Delta \mathrm{FC}$ (Figure 7) showed that the mean error of $14.7 \%$ was a reasonable estimate of the overall error in FC $(\triangle \mathrm{FC})$ caused by HRF variability. For weaker connection strengths, this number can reach up to $170 \%$ (Figure 3D). We observed HRF variability to have a widespread impact even among those connections not considered significantly different. Still, these numbers did not correspond to the worst case because they were obtained from data with outliers removed. The outliers $(12.9 \%$ in our data), corresponding to large percentage changes in FC, exist in any data and likely impact any FC analysis performed using it. If outliers were included, we observed average connectivity error of $30.5 \%$; however, to be conservative, we chose to focus on results after outlier elimination in this study even though it is conceivable that outliers may be connections of interest in certain scenarios. These findings imply that the combined effect of HRF parameters on FC in experimental data is at least as worse as the simulation results, and HRF variability, on average, causes $\sim 14.7 \%$ error in the FC data in healthy controls for the scan parameters we have used in a 7T scanner. It is not possible to unbundle the effects of each HRF parameter on $\triangle \mathrm{FC}$, because, in experimental data, $\triangle F C$ has contributions from a variable mix of all 3 HRF parameters. It is important to acknowledge that an error of the order of $15 \%$ in connectivity estimates is sufficient to cause a large impact on statistical inferences, network structure, graph analysis, machine learning models, and any other derived measure such as behavioral associations. Even in psychiatric and neurologic disorders, aberrant changes in connectivity values are often of this order or smaller. $^{34}$

To supplement these observations, we fit a double exponential curve (Figure 8) that quantifies the relationship between HRF variability and its resultant impact on FC using experimental data. Among several examples provided in the previous section, 2 examples that stood out were that onethird of all the connectivities changed by over $10 \%$ after deconvolution, and every tenth connection changed by as much as one-third after deconvolution. Even if one were to question the validity of our deconvolution approach and argue that the ground-truth HRF would be less variable than our estimated HRFs, our findings from the "bottom 25\%" analysis suggests that comparably strong effects persist even if one were to hypothesize less variable and "better quality" unknown ground-truth HRFs. In view of this, we argue that the conclusions we have drawn in this study are robustly applicable to the study of FC using resting-state BOLD fMRI. Given that the effect sizes we often observe with fMRI are relatively small, these numbers, which are by no means ignorable, illustrate the considerable impact that deconvolution has on BOLD fMRI data.

While interpreting these numbers, it must be acknowledged that our analysis was limited to the DMN, was in a homogenous healthy adult cohort, and data was obtained in a 7T scanner. HRF variability would be higher across the entire brain compared to the DMN only, would be higher in a disease group or in different age/race/gender groups, and would be higher at poorer sampling rates or smaller field strengths. ${ }^{43}$ It is noteworthy that pathological populations are likely to have a wider physiologically plausible range of 
HRF parameters because of neurochemical and vascular alterations, and hence, likely larger confounds in FC because of HRF variability. We posit that the mean connectivity error or $\Delta \mathrm{FC}$ would be much higher in such cases. In view of this, we feel that the readers should consider the worst-case impact of HRF variability to be likely worse than our results. We invite researchers to perform similar studies under different conditions to help develop a broader understanding of the problem.

An error in connectivity values by as much as $15 \%$ in a best-case scenario demands serious attention. Such large errors and such shifts in average connectivity values can bias statistical results when comparing 2 different groups of participants. If the shift in connectivity is away from the group mean of the other group, it could falsely increase statistical separation between the groups, resulting in increased $T$-values, $F$-values, or any other statistic, leading to the possible identification of false positives. On the other hand, a shift of connectivity toward the group mean of the other group could inadvertently reduce statistical separation between the groups, resulting in decreased $T$-values or $F$-values, leading to possible false negatives. During group-comparisons, such phenomena, in the best-case scenario, would alter the $T$-values, $F$-values, $P$-values, or other statistical metrics, resulting in biased results. In the worst-case scenario, it would cause the identification of false connections or result in missed connections. It could have enormous impact while performing group comparisons in population studies because the effect sizes often encountered in brain imaging are small. Further, the effect size of the impact of HRF variability on FC might be larger in patient populations, given that many of them are likely to have vascular and/or neurochemical alterations, more than that observed in a healthy population.

Given these findings, future fMRI studies must exercise caution while interpreting results obtained from resting-state FC analysis of non-deconvolved BOLD fMRI data, especially if they assume a fixed canonical HRF. Although only a handful to regions showed HRF differences in this work, it does not imply that HRF variability does not exist elsewhere, because we used a conservative statistical threshold that might have ignored smaller effects. Additionally, it has been recognized that fMRI signals at ultrahigh fields have more contributions from small vessels, ${ }^{43}$ because the variability of HRF is likely less for small vessels at higher field strengths. Hence, the HRF confound in our data may be less severe than in data acquired at lower fields, such as 3T. Nonetheless, even with our data from a 7T MRI scanner, we show that the HRF variability is a major cause of error in estimated functional connectivities. Such errors are expected to be larger for data obtained at 3T.

Prior works have studied the impact of HRF variability on modeling the mean of the fMRI time series, ${ }^{2,3,44}$ whereas our novelty rests on studying the impact of HRF variability on modeling FC. We found erroneous connectivities emerging because of intra-subject HRF variability, which corroborates with the fact that these prior studies found HRF variability across different brain regions within an individual. Our work is significant given that the impact of HRF variability on FC has not been formally established in literature yet, with the consequence that a large number of FC studies continue to ignore the confound of HRF variability in their findings. We hope that our study encourages researchers to overcome this perplexing lack of attention to HRF variability while estimating resting-state fMRI FC. Literature on fMRI FC has been increasing exponentially (1435 publications in 2016 alone and 1241 in 2017 up to September), yet an awareness of the confound of HRF variability has not emerged, and almost all studies ignore HRF variability in their analysis.

The focus of this work was primarily to identify the impact of HRF variability on FC at the individual-subject level (or intra-subject variability) in a healthy population. Intra-group inter-subject variability and inter-group variability could be topics of future investigation. Such studies have begun to emerge with recent papers reporting confounds in inter-group resting-state FC differences in a cohort of soldiers with and without PTSD (post-traumatic stress disorder) and mTBI (mild traumatic brain injury). ${ }^{5,28}$ Future studies could also study the effect of HRF variability on FC at the wholebrain level in the healthy brain, as well as characterize the impact of HRF variability in specific psychiatric disorders.

Although the HRF is not grounded purely on neural activity, it is worthwhile to note that it is not useless noise, but an information-carrying biological measurement. ${ }^{5} \mathrm{HRF}$ estimates (using fMRI) are not direct biophysical measurements, much like other derived fMRI measures (e.g., connectivity, activations), hence some degree of uncertainty (as estimates) is associated with them. With this limitation in the background, we encourage researchers to use hemodynamic deconvolution during data pre-processing to minimize (even if it may not be possible to completely eliminate) the impact of HRF variability on fMRI connectivity modeling. Our findings are not limited to the deconvolution technique used in this work, and researchers could use other established deconvolution techniques ${ }^{45-48}$ to likely arrive at similar conclusions. Finally, it is notable that unlike RH, fMRI temporal resolution significantly matters for accurate TTP and FWHM estimation, with their resolution limited by acquisition repetition time (TR). With the recent advent of fast fMRI techniques, data can be acquired with TRs of up to $400 \mathrm{~ms}$ compared to a TR of $1 \mathrm{~s}$ used in our study. We recommend future studies focusing on $\mathrm{HRF}$ analysis to acquire data at finer temporal resolution.

\section{5 | CONCLUSIONS}

Functional MRI being an indirect measure of neural activity, non-neural factors must be carefully accounted for while 
interpreting fMRI findings. These non-neural factors express through variability in HRF across brain regions and subjects, rendering fMRI findings to be less reliable. Using both simulations (where the ground truth is known) as well as wholebrain resting-state fMRI from a 7T MRI scanner, we tested the hypothesis that such HRF variability causes false functional connections to be inferred. We found evidence in support of our hypothesis. With simulations, we found that $\mathrm{RH}$ and TTP could independently cause a change of up to 50\% in FC, whereas FWHM could cause about 5\% change. With experimental data, we found significantly different FC between deconvolved and non-deconvolved data, with HRF parameters also being significantly different between the corresponding regions, therefore attributing the altered connectivities to undesirable HRF variability. On average, HRF variability resulted in an error of $14.7 \%$ in FC using a bestcase scenario where outliers are eliminated. Several pseudopositive and pseudo-negative connections were found within the DMN, with more pseudo-positives identified. Pseudopositives are more detrimental to fMRI analysis than pseudonegatives. In addition, most of the connections were between different lobes, owing to the fact that the underlying neurochemistry and vascular structure are more heterogeneous across distant and distinct regions. We showed that even a less noisy and less variable HRF could lead to considerable confounds in FC estimates. We conclude that HRF variability could cause potentially false functional connections to be identified in the brain. To the best of our knowledge, this is the first systematic study on the impact of HRF variability on resting-state $\mathrm{FC}$ in healthy individuals. These findings have enormous implications in the analysis and interpretation of fMRI data. FC findings from non-deconvolved data must be interpreted with caution. Researchers are encouraged to perform hemodynamic deconvolution during pre-processing to minimize HRF variability. The deconvolution code and all data associated with this article are made publicly available. ${ }^{34}$ In the future, we plan to publish a user-interfacebased toolbox to perform deconvolution.

\section{ORCID}

D. Rangaprakash (10) http://orcid.org/0000-0001-9553-1354

Daniele Marinazzo (1) http://orcid.org/0000-0002-9803-0122

Gopikrishna Deshpande (D) http://orcid.org/0000-0001-74715357

\section{REFERENCES}

[1] Len TK, Neary JP. Cerebrovascular pathophysiology following mild traumatic brain injury. Clin Physiol Funct Imaging. 2011; 31:85-93.

[2] Handwerker DA, Ollinger JM, D'Esposito M. Variation of BOLD hemodynamic responses across subjects and brain regions and their effects on statistical analyses. Neuroimage. 2004;21: 1639-1651.

[3] Aguirre GK, Zarahn E, D'esposito M. The variability of human, BOLD hemodynamic responses. Neuroimage. 1998;8:360-369.

[4] Wu GR, Marinazzo D. Sensitivity of the resting-state haemodynamic response function estimation to autonomic nervous system fluctuations. Philos Trans A Math Phys Eng Sci. 2016;374.

[5] Rangaprakash D, Dretsch MN, Yan W, Katz JS, Denney TS Jr, Deshpande G. Hemodynamic variability in soldiers with trauma: implications for functional MRI connectivity studies. Neuroimage Clin. 2017;16:409-417.

[6] Hall CN, Howarth C, Kurth-Nelson Z, Mishra A. Interpreting BOLD: towards a dialogue between cognitive and cellular neuroscience. Philos Trans R Soc Lond B Biol Sci. 2016;371.

[7] Buckner RL, Andrews-Hanna JR, Schacter DL. The brain's default network: anatomy, function, and relevance to disease. Ann N Y Acad Sci. 2008;1124:1-38.

[8] Poldrack RA, Mumford JA, Nichols TE. Handbook of functional MRI data analysis. New York: Cambridge University Press; 2011. 238 p.

[9] Deshpande G, Sathian K, Hu X. Effect of hemodynamic variability on Granger causality analysis of fMRI. Neuroimage. 2010;52:884-896.

[10] Handwerker DA, Gonzalez-Castillo J, D'Esposito M, Bandettini PA. The continuing challenge of understanding and modeling hemodynamic variation in fMRI. Neuroimage. 2012;62:1017-1023.

[11] Buxton R. Introduction to functional magnetic resonance imaging: principles and techniques. New York: Cambridge University Press; 2002. 523 p.

[12] Noseworthy MD, Alfonsi J, Bells S. Attenuation of brain BOLD response following lipid ingestion. Hum Brain Mapp. 2003;20: 116-121.

[13] Friston KJ, Harrison L, Penny W. Dynamic causal modelling. Neuroimage. 2003;19:1273-1302.

[14] Mayer AR, Toulouse T, Klimaj S, Ling JM, Pena A, Bellgowan PS. Investigating the properties of the hemodynamic response function after mild traumatic brain injury. J Neurotrauma. 2014; 31:189-197.

[15] Deshpande G, Sathian K, Hu X. Assessing and compensating for zero-lag correlation effects in time-lagged Granger causality analysis of FMRI. IEEE Trans Biomed Eng. 2010;57:1446-1456.

[16] Friston K, Ashburner J, Kiebel S, Nichols T, Penny W. Statistical parametric mapping: the analysis of functional brain images. Amsterdam: Academic Press; 2007. 656 p.

[17] Roebroeck A, Formisano E, Goebel R. Mapping directed influence over the brain using Granger causality and fMRI. Neuroimage. 2005;25:230-242.

[18] Logothetis NK, Pauls J, Augath M, Trinath T, Oeltermann A. Neurophysiological investigation of the basis of the fMRI signal. Nature. 2001;412:150-157.

[19] Feinberg DA, Moeller S, Smith SM, et al. Multiplexed echo planar imaging for sub-second whole brain fMRI and fast diffusion imaging. PLoS One. 2010;5:e15710.

[20] Power JD, Cohen AL, Nelson SM, et al. Functional network organization of the human brain. Neuron. 2011;72:665-678. 
[21] Wu GR, Liao W, Stramaglia S, Ding JR, Chen H, Marinazzo D. A blind deconvolution approach to recover effective connectivity brain networks from resting state fMRI data. Med Image Anal. 2013;17:365-374.

[22] Amico E, Gomez F, Di Perri C. Posterior cingulate cortexrelated co-activation patterns: a resting state FMRI study in propofol-induced loss of consciousness. PLoS One. 2014;9: e100012.

[23] Boly M, Sasai S, Gosseries O, et al. Stimulus set meaningfulness and neurophysiological differentiation: a functional magnetic resonance imaging study. PLoS One. 2015;10:e125337.

[24] Lamichhane B, Adhikari BM, Brosnan SF, Dhamala M. The neural basis of perceived unfairness in economic exchanges. Brain Connect. 2014;4:619-630.

[25] Liang P, Deshpande G, Zhao S, Liu J, Hu X, Li K. Altered directional connectivity between emotion network and motor network in Parkinson's disease with depression. Medicine (Baltimore). 2016;95:e4222.

[26] Rangaprakash D, Deshpande G, Daniel TA, et al. Compromised hippocampus-striatum pathway as a potential imaging biomarker of mild traumatic brain injury and posttraumatic stress disorder. Hum Brain Mapp. 2017;38:2843-2864.

[27] Rangaprakash D, Dretsch MN, Venkataraman A, Katz JS, Denney TS Jr, Deshpande G. Identifying disease foci from static and dynamic effective connectivity networks: illustration in soldiers with trauma. Hum Brain Mapp. 2018;39:264-287.

[28] Rangaprakash D, Dretsch MN, Yan W, Katz JS, Denney TS Jr, Deshpande G. Hemodynamic response function parameters obtained from resting-state functional MRI data in soldiers with trauma. Data Brief. 2017;14:558-562.

[29] Wu GR, Marinazzo D. Point-process deconvolution of fMRI bold signal reveals effective connectivity alterations in chronic pain patients. Brain Topogr. 2015;28:541-547.

[30] Power JD, Schlaggar BL, Petersen SE. Recent progress and outstanding issues in motion correction in resting state fMRI. Neuroimage. 2015;105:536-551.

[31] Tagliazucchi E, Balenzuela P, Fraiman D, Chialvo DR. Criticality in large-scale brain fMRI dynamics unveiled by a novel point process analysis. Front Physiol. 2012;3:15.

[32] Marinazzo D. Code for HRF blind deconvolution. 2013. http:// users.ugent.be/ dmarinaz/HRF_deconvolution.html. Accessed September 2016.

[33] Rangaprakash D, Wu GR, Marinazzo D, Hu X, Deshpande G. Parametrized hemodynamic response function data of healthy individuals obtained from resting-state functional MRI in a 7T MRI scanner. Data Brief. 2018. https://doi.org/10.1016/j.dib. 2018.01.003.

[34] Gross J. Handbook of emotion regulation. New York: The Guilford Press; 2014. 654 p.

[35] Thompson SK, Engel SA, Olman CA. Larger neural responses produce BOLD signals that begin earlier in time. Front Neurosci. 2014;8:159.

[36] Buzsáki G, Kaila K, Raichle M. Inhibition and brain work. Neuron. 2007;56:771-783.

[37] Brown GG, Eyler Zorrilla LT, Georgy B, Kindermann SS, Wong EC, Buxton RB. BOLD and perfusion response to finger- thumb apposition after acetazolamide administration: differential relationship to global perfusion. J Cereb Blood Flow Metab. 2003;23:829-837.

[38] Muthukumaraswamy SD, Evans CJ, Edden RA, Wise RG, Singh $\mathrm{KD}$. Individual variability in the shape and amplitude of the BOLD-HRF correlates with endogenous GABAergic inhibition. Hum Brain Mapp. 2012;33:455-465.

[39] Busija DW, Bari F, Domoki F, Louis T. Mechanisms involved in the cerebrovascular dilator effects of N-methyl-d-aspartate in cerebral cortex. Brain Res Rev. 2007;56:89-100.

[40] Cohen Z, Bonvento G, Lacombe P, Hamel E. Serotonin in the regulation of brain microcirculation. Prog Neurobiol. 1996;50: 335-362.

[41] Hillman EM. Coupling mechanism and significance of the BOLD signal: a status report. Annu Rev Neurosci. 2014;37:161-181.

[42] Murphy MC, Chan KC, Kim SG, Vazquez AL. Macroscale variation in resting-state neuronal activity and connectivity assessed by simultaneous calcium imaging, hemodynamic imaging and electrophysiology. Neuroimage. 2017;169:352-362.

[43] Yacoub E, Shmuel A, Pfeuffer J, et al. Imaging brain function in humans at 7 Tesla. Magn Reson Med. 2001;45:588-594.

[44] Lindquist MA, Meng Loh J, Atlas LY, Wager TD, Modeling the hemodynamic response function in fMRI: efficiency, bias and mis-modeling. Neuroimage. 2009;45(1Suppl):S187-S198.

[45] Havlicek M, Friston KJ, Jan J, Brazdil M, Calhoun VD. Dynamic modeling of neuronal responses in fMRI using cubature Kalman filtering. Neuroimage. 2011;56:2109-2128.

[46] Sreenivasan KR, Havlicek M, Deshpande G. Nonparametric hemodynamic deconvolution of FMRI using homomorphic filtering. IEEE Trans Med Imaging. 2015;34:1155-1163.

[47] Aquino KM, Robinson PA, Schira MM, Breakspear M. Deconvolution of neural dynamics from fMRI data using a spatiotemporal hemodynamic response function. Neuroimage. 2014;94: 203-215

[48] Bush K, Cisler J, Bian J, Hazaroglu G, Hazaroglu O, Kilts C. Improving the precision of fMRI BOLD signal deconvolution with implications for connectivity analysis. Magn Reson Imaging. 2015;33:1314-1323.

\section{SUPPORTING INFORMATION}

Additional Supporting Information may be found in the online version of this article.

FIGURE S1 Simulation results for empirical assessment of percentage change in functional connectivity $(\Delta \mathrm{FC})$ between neural and BOLD time series caused by difference in HRF parameters ( $\Delta$ RH, $\Delta T T P, \Delta F W H M$ ) between the corresponding 2 time series, across all possible physiologically plausible values of these HRF measures. The results are presented for 4 different sampling rate (TR) values: $0.5,1,1.5$, and $2 \mathrm{~s}$. A similar figure in the main document (Figure 3) presented results only for $\mathrm{TR}=1 \mathrm{~s}$. (A) $\Delta \mathrm{FC}$ versus $\Delta \mathrm{RH}(\mathrm{RH}$ resolution $=0.25 \%)$. (B) $\Delta \mathrm{FC}$ versus $\Delta \mathrm{TTP}(\mathrm{TTP}$ resolution $=0.25 \mathrm{~s})$. $(\mathrm{C}) \Delta \mathrm{FC}$ versus $\Delta \mathrm{FWHM}$ 
$(\mathrm{FWHM}$ resolution $=0.25 \mathrm{~s})$. (D) $\Delta \mathrm{FC}$ versus absolute value of neural FC $(\mathrm{FC}$ resolution $=0.25)$. Error bars show $1 \mathrm{SD}$ above and below the mean. Figure-S1a does not show error bars because the error bars would mask the mean curve if used; nonetheless, the SDs are nearly identical to the ones visible in Figure 3A. The physiologically plausible lower and upper bounds of HRF parameters were obtained from Handwerker et al. ${ }^{2}$ There was no significant impact of TR, except with FWHM at $1 \mathrm{~s}$ (corrected $P<0.05)$. We observed robust and nearly linear positive relationship between $\triangle F C$ and change in HRF parameters. Up to $50 \%$ change in FC was observable because of either RH or TTP variability. Also smaller FC values were more vulnerable to HRF variability. RH, response height; TTP, time-to-peak; FWHM, full-width at half-max; FC, functional connectivity; TR, repetition time

FIGURE S2 Comparing the frequency spectra (power spectral density) of deconvolved and non-deconvolved data, obtained from the entire data set. (A) Mean spectra: we noticed them to be nearly overlapping. (B) Spectra with SDs, along with the zoomed inlet showing the location of $-20 \mathrm{~dB}$ upper cut-off power (100 times attenuation with respect to the peak). We can observe that the SDs were large compared to mean difference at that point, which is why they were not significant $(P>0.05)$. The two spectra were found to not differ
TABLE S1 The 58 ROIs of the default mode network used in this work, which was adopted from Power et al.21 The MNI coordinates of each ROI are provided, along with ROI name obtained from Talairach Daemon (http:// www.talairach.org/daemon.html). Each ROI was defined as a sphere of radius $5 \mathrm{~mm}$ around the centroid of each ROI. Averaged time series obtained from all voxels in each ROI were used for further analysis

TABLE S2 Significant connections $(P<0.05$, Bonferroni corrected) with functional connectivity being higher with deconvolved data compared to non-deconvolved data (pseudo negatives). The MNI coordinates and names of the corresponding ROIs can be obtained from Table S1

TABLE S3 Significant connections $(P<0.05$, Bonferroni corrected) with functional connectivity being higher with non-deconvolved data compared to deconvolved data (pseudo-positives). The MNI coordinates and names of the corresponding ROIs can be obtained from Table S1

How to cite this article: Rangaprakash D, Wu G-R, Marinazzo D, Hu X, Deshpande G. Hemodynamic response function (HRF) variability confounds restingstate fMRI functional connectivity. Magn Reson Med. 2018;00:1-17. https://doi.org/10.1002/mrm.27146 